\title{
Chirality Selection in Crystallization
}

\author{
Yukio SAITO* and Hiroyuki HyugA ${ }^{\dagger}$ \\ Department of Physics, Keio University, Yokohama 223-8522
}

(Received July 19, 2017)

\begin{abstract}
A cluster growth model is proposed to study chiral symmetry breaking in stirred crystallization from an achiral element. Achiral monomers are assumed to coagulate to form chiral clusters from dimers to hexamers. Due to the stirring, the hexamers break into dimers. The coagulation of two dimers into a tetramer also occurs. The fixed point analysis of coupled rate equations of cluster densities and their numerical integrations show that the chiral symmetry becomes broken if dimers are critical and able to dissociate back to achiral monomers. The chirality selection is understood by showing that, in a quasi-steady approximation, the rate equations reduce to those of dimers with nonlinear autocatalysis and decomposition.

KEYWORDS: homochirality, crystal growth, enantiomer, rate equation, dynamical chiral symmetry breaking
\end{abstract}

It has been known for a long time that organic molecules in life are homochiral with a completely broken chiral symmetry, and their origin has intrigued many scientists. ${ }^{1}$ Frank showed theoretically that an autocatalytic reaction with cross inhibition between two enatiomers leads to the selection of one type of enantiomer in an open system. ${ }^{2}$ We have recently proven that a system with nonlinear autocatalysis and back reaction as is described by the following rate equations

$$
\begin{aligned}
& \dot{r}=\left(k_{0}+k_{1} r+k_{2} r^{2}\right) a-\lambda r, \\
& \dot{s}=\left(k_{0}+k_{1} s+k_{2} s^{2}\right) a-\lambda s,
\end{aligned}
$$

can lead to complete homochirality in a closed system. ${ }^{3-5}$ Here, $a$ denotes the concentration of an achiral substrate, $r$ and $s$ are those of chiral enantiomers, and the conservation relation $a+r+s=$ const. is assumed to hold. The model is motivated by the chemical reaction system discovered by Soai et al., ${ }^{6,7}$ which is the first example of chemical reaction to show the amplification of enantiomeric excess in a closed system.

Chiral symmetry breaking, however, is found not only in biological and chemical systems, but also in other systems such as in crystallization. ${ }^{8}$ Even achiral inorganic molecules can gather together to form chiral crystals. Examples are a quartz of $\mathrm{SiO}_{2}$ crystal, or a crystal of sodium chlorate $\left(\mathrm{Na} \mathrm{ClO}_{3}\right)$. When the sodium chlorate crystal grows from a solution in a cell, a racemic mixture of $D$ and $L$ crystals emerges. On the other hand, when the crystal is

\footnotetext{
*yukio@rk.phys.keio.ac.jp

†hyuga@rk.phys.keio.ac.jp
} 
grown from a supersaturated solution under continuous stirring, the growth cell is occupied by one type of enantiomer. ${ }^{9}$ The chirality selection is found to be brought about by fragmenting an initially nucleated crystal: the scattered clusters of small crystal act as centers of secondary nucleation of the same chirality as that of the initial crystal. This senario is called a " single mother" crystallization. Recently, however, it has been found that even if the initial solution contains crystalline seeds of both enantiomers, the continuous stirring leads to the state of complete chiral purity with one type of enantiomer. The selected enantiomer is the one which was in a slight excess initially. ${ }^{10}$ There is a theoretical study associated with this phenomenon, ${ }^{11}$ but we propose another approach in order to examine the roles of various elementary processes. We also study the relationship between the present model and eq. (1) reported in our previous studies..$^{3,5}$

Our model of crystallization is described in terms of clusters of various sizes. An individual molecule $\mathrm{A}$ is achiral, but a few of them coagulate into a chiral embryo. For simplicity, we assume here that dimers already show the chirality. We call the right-handed dimer $\mathrm{R}_{2}$, and the left-handed one $\mathrm{S}_{2}$. The first growth process is then described by

$$
A+A \underset{\lambda}{\stackrel{k_{1}}{\rightleftharpoons}} R_{2}, \quad A+A \underset{\lambda}{\stackrel{k_{1}}{\rightleftharpoons}} S_{2} .
$$

These chiral embryos grow further by incoorporating elements A one by one as,

$$
R_{i}+A \stackrel{k_{i}}{\rightarrow} R_{i+1}, \quad S_{i}+A \stackrel{k_{i}}{\rightarrow} S_{i+1},
$$

with $i \geq 2$. Here, we assume for simplicity that the critical size of the crystal is $i=2$. Only the dimers can dissociate with a rate $\lambda$, which is assumed larger than the growth rate $k_{1}$ from monomers into a dimer. Clusters larger than the dimer are stable and only grow. If these are the whole processes, the crystalline enantiomeric excess does not increase. In the experiment ${ }^{10}$ the solution is continuously stirred by a stirrer and balls, ${ }^{10}$ which crash part of the grown crystals and split off small clusters.

First we consider the case that the smallest nontrivial cluster, tetramer, splits as

$$
R_{4} \stackrel{\nu}{\rightarrow} R_{2}+R_{2}, \quad S_{4} \stackrel{\nu}{\rightarrow} S_{2}+S_{2}
$$

with a rate $\nu$. Coupled rate equations for concentrations $r_{i}, s_{i}$ of dimers $(i=2)$, trimers $(i=3)$ and tetramers $(i=4)$ are written as

$$
\begin{aligned}
& \dot{r}_{2}=k_{1} a^{2}-\lambda r_{2}-k_{2} r_{2} a+2 \nu r_{4} \\
& \dot{r}_{3}=k_{2} r_{2} a-k_{3} r_{3} a \\
& \dot{r}_{4}=k_{3} r_{3} a-\nu r_{4}
\end{aligned}
$$

and the corresponding ones for S's. Since the total amount of the molecules is conserved in a closed reaction vessel, the concentration of elementary molecule A is given as $a=c_{0}-2\left(r_{2}+\right.$ $\left.s_{2}\right)-3\left(r_{3}+s_{3}\right)-4\left(r_{4}+s_{4}\right)$ with a constant $c_{0}$. Numerical integration indicates readily that the 
chiral symmetry will not be broken in the present case. This result can be easily undestood by applying the quasi-steady state approximation. Because of large reaction rates $k_{i}(i \geq 2)$ and $\nu$, clusters larger than the dimers are assumed to adjust their concentrations rapidly to their steady-state values as $r_{3}=\left(k_{2} / k_{3}\right) r_{2}, r_{4}=\left(k_{2} / \nu\right) r_{2} a$, as long as $a \neq 0$. Then the evolution of the dimer concentrations, $r_{2}$ and $s_{2}$, is simply described by the reduced rate equations

$$
\begin{aligned}
& \dot{r}_{2}=k_{1} a^{2}-\lambda r_{2}+k_{2} a r_{2}, \\
& \dot{s}_{2}=k_{1} a^{2}-\lambda s_{2}+k_{2} a s_{2} .
\end{aligned}
$$

The equation contains only a linear autocatalytic process and a back reaction, and looks similar to eq. (1) with $k_{2}=0 .{ }^{3} \mathrm{~A}$ simple calculation shows that there is only a racemic fixed point as $r_{2}=s_{2}=k_{1} a^{2} /\left(\lambda-k_{2} a\right)$. Therefore, the system approaches a racemic state asymptotically, in agreement with numerical integration results. The chiral symmetry is conserved. The key point is that the secondary nucleation induces the autocatalytic effect in the dimer evolution, but only up to the level of linear autocatalysis. Note that the fragmentation effect $\nu$ does not appear explicitly in the reduced rate equation (6).

Since the chirality is not selected in the cluster growth up to the tetramer, we go one step further, up to the hexamer; $\mathrm{R}_{i}, \mathrm{~S}_{i}$ with $2 \leq i \leq 6$. Instead of eq. (4), we assume the breaking of a hexamer into three dimers as

$$
R_{6} \stackrel{\nu}{\rightarrow} 3 R_{2}, \quad S_{6} \stackrel{\nu}{\rightarrow} 3 S_{2},
$$

and furthermore the incorporation of the coagulation of dimers into a tetramer

$$
R_{2}+R_{2} \stackrel{\mu}{\rightarrow} R_{4}, \quad S_{2}+S_{2} \stackrel{\mu}{\rightarrow} S_{4}
$$

Then the rate equations are written as

$$
\begin{aligned}
& \dot{r}_{2}=k_{1} a^{2}-\lambda r_{2}-k_{2} r_{2} a-2 \mu r_{2}^{2}+3 \nu r_{6} \\
& \dot{r}_{3}=k_{2} r_{2} a-k_{3} r_{3} a \\
& \dot{r}_{4}=k_{3} r_{3} a-k_{4} r_{4} a+\mu r_{2}^{2} \\
& \dot{r}_{5}=k_{4} r_{4} a-k_{5} r_{5} a \\
& \dot{r}_{6}=k_{5} r_{5} a-\nu r_{6}
\end{aligned}
$$

and the corresponding ones for S's, under the conservation condition $a=c_{0}-\sum_{i=2}^{6} i\left(r_{i}+s_{i}\right)$.

Since these coupled equations involve many degrees of freedom, detailed analysis can be performed only by numerical integration. However, the essential feature may be understood in a quasi-steady approximation as follows. By assuming the rapid variation of concentrations of clusters larger than the critical size $i=2$, their concentrations are approximated by those values determined by the given concentrations of the critical cluster as; $r_{3}=$ $\left(k_{2} / k_{3}\right) r_{2}, r_{4}=\left(k_{2} / k_{4}\right) r_{2}+\left(\mu / k_{4} a\right) r_{2}^{2}, r_{5}=\left(k_{2} / k_{5}\right) r_{2}+\left(\mu / k_{5} a\right) r_{2}^{2}, r_{6}=\left(k_{2} / \nu\right) r_{2} a+(\mu / \nu) r_{2}^{2}$, 
and the corresponding relations for $s$ 's, as long as $a \neq 0$. In particular, we obtain the relation $\nu r_{6}=k_{2} r_{2} a+\mu r_{2}^{2}$. By inserting the relation into the rate equations of the dimers, one obtains the reduced rate equations for the dimers as

$$
\begin{aligned}
& \dot{r}_{2}=k_{1} a^{2}-\lambda r_{2}+2 k_{2} r_{2} a+\mu r_{2}^{2}, \\
& \dot{s}_{2}=k_{1} a^{2}-\lambda s_{2}+2 k_{2} s_{2} a+\mu s_{2}^{2} .
\end{aligned}
$$

These equations contain essential terms such as the decomposition term $-\lambda r_{2}$ (or $\left.-\lambda s_{2}\right)$ and a nonlinear autocatalytic term $\mu r_{2}^{2}$ ( or $\mu s_{2}^{2}$ ). In this sense we expect that eq. (10) shows similar characteristics to that of eq. (1). For example, if the dissociation is absent, $\lambda=0$, then $\dot{r}_{2}$ and $\dot{s}_{2}$ consist only of positive terms and the stationary solution $\dot{r}_{2}=\dot{s}_{2}=0$ is possible only for $a=r_{2}=s_{2}=0$. From the stationary condition for hexamers we obtain $r_{6}=s_{6}=0$, but other concentrations $r_{i}$ and $\mathrm{s}_{i}$ with $3 \leq i \leq 5$ are arbitrary as long as the conservation relation is satisfied. No chirality selection is possible, since there are no fixed points, but fixed lines or planes.

We now study the case with dissociation $(\lambda>0)$. One notices that the fixed points for the full rate equation (9) are identical with those obtained by the reduced rate equation (10) by way of the construction of the latter. There are racemic fixed points $\left(r_{2}=s_{2}\right)$ which are not of our interest. As for the asymmetric fixed points, it can be easily shown that they should satisfy the relations

$$
\begin{aligned}
& r_{2}+s_{2}=\frac{\lambda-2 k_{2} a}{\mu}>0, \quad r_{2} s_{2}=\frac{k_{1} a^{2}}{\mu}, \\
& c_{0}=a+(A+B a) k_{2}\left(r_{2}+s_{2}\right)+(C / a+B) \mu\left(r_{2}^{2}+s_{2}^{2}\right),
\end{aligned}
$$

with $A=\sum_{i=2}^{5} i k_{i}^{-1}, B=6 / \nu, C=\sum_{i=4}^{5} i k_{i}^{-1}$. The first two relations of eq. (11) yield two asymmetric fixed points $\mathrm{S}_{1}:\left(r_{2,+}, s_{2,-}\right)$ and $\mathrm{S}_{2}:\left(r_{2,-}, s_{2,+}\right)$ with

$$
r_{2, \pm}=\frac{\lambda-2 k_{2} a}{2 \mu} \pm \sqrt{\left(\frac{\lambda-2 k_{2} a}{2 \mu}\right)^{2}-\frac{k_{1} a^{2}}{\mu}}=s_{2, \mp},
$$

which is expressed by using a fixed point value of $a$ yet to be determined. For the existence of these asymmetric fixed points, $a$ should be in the range $\lambda / 2\left(k_{2}+\sqrt{\mu k_{1}}\right) \geq a>0$. Finally the asymmetric fixed points are determined by solving an algebraic equation of $a$, obtained by inserting the first two relations in eq. (11) into the last one.

Since the conditions for the existence of the asymmetric fixed points are very involved, we restrict the discussion here to the case of $c_{0}=k_{2}=k_{3}=k_{4}=k_{5}=1$. Then it can be shown that the asymmetric fixed points exist for $\lambda$ 's in the range

$$
0<\lambda<\lambda_{u}
$$

where the upper limit $\lambda_{u}$ is given by the larger root of the quadratic equation

$$
6 \beta \lambda^{2}+\nu\left(\mu+28 \beta+18 \beta^{2}\right) \lambda-2 \mu \nu(1+\beta)=0
$$


with $\beta=\sqrt{\mu k_{1}}$. In particular, $\lambda_{u} \simeq 2$ for $k_{1} \simeq 0$ and $\lambda_{u} \simeq \mu \nu / 9 \beta \rightarrow 0$ for $k_{1} \rightarrow \infty$.

We then perform numerical integration of the rate equation (9), in order to observe the chiral symmetry breaking in the crystallization. Since the formation of a critical nucleus (=a dimer) is expected to be slow, $k_{1}$ is set as small as $k_{1}=0.01$ compared with other parameters as $c_{0}=1, k_{2}=k_{3}=k_{4}=k_{5}=1, \lambda=0.3, \mu=\nu=1$. The decomposition rate $\lambda=0.3$ is set smaller than the upper limit $\lambda_{u}=0.513$ calculated from eq. (14), and one of the asymmetric fixed points is expected to be realized. This expected behavior is in fact observed in the flow diagram shown in Fig. 1 in the phase space of the total number densities of $\mathrm{R}$ and $\mathrm{S}$ crystals, $r_{\Sigma}=\sum_{i=2}^{6} i r_{i}$ and $s_{\Sigma}=\sum_{i=2}^{6} i s_{i}$, respectively. By starting close to the symmetric mixture $r_{\Sigma} \approx s_{\Sigma}$, both enantiomeric crystals increase in number, approaching the racemic fixed point, but this racemic fixed point is unstable, and eventually one enantiomer overwhelms the other. The flow is attracted to one of the chiral fixed points. If one decreases $k_{1}$, the chiral asymmetry represented by the crystalline enantiomeric excess cee $=\left|\left(r_{\Sigma}-s_{\Sigma}\right) /\left(r_{\Sigma}+s_{\Sigma}\right)\right|$ increases up to almost unity.

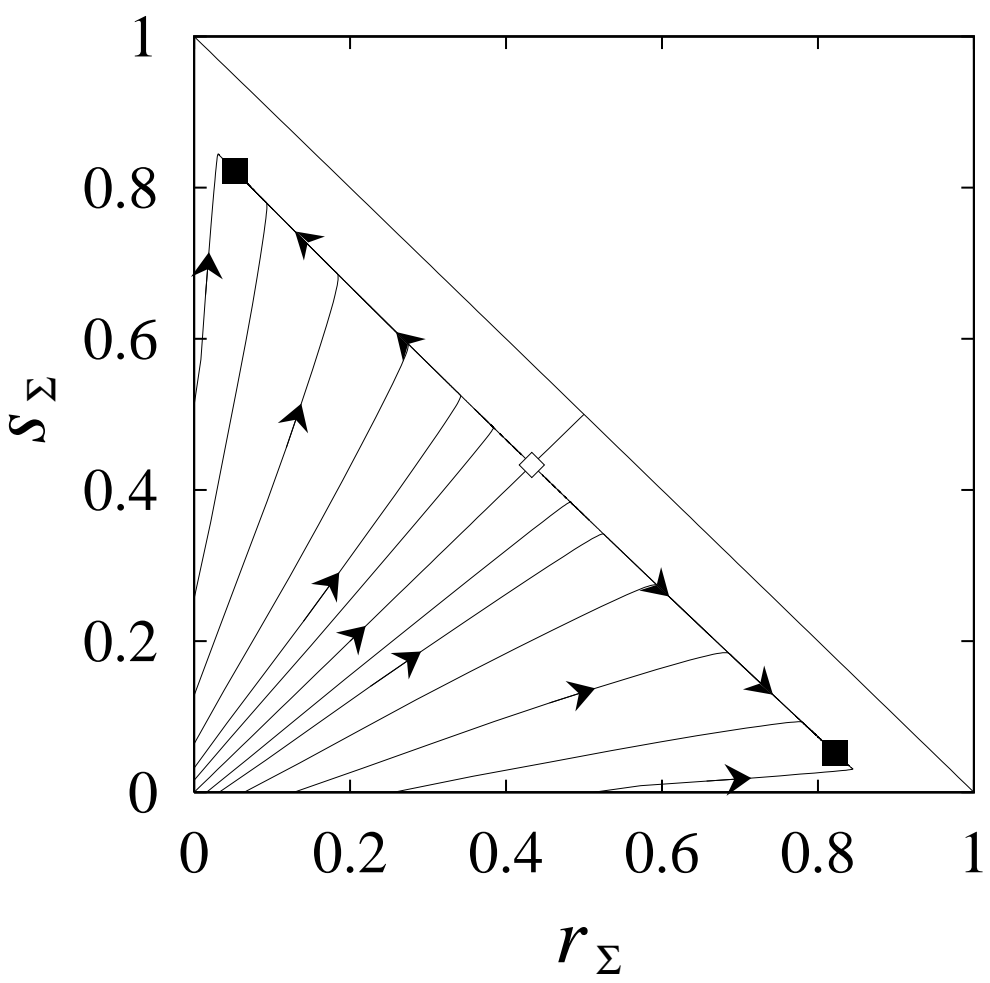

Fig. 1. Flow diagram in total $r$ 's and $s$ 's phase space. Symbols represents symmetric and asymmetric fixed points. The parameters are $c_{0}=1, k_{1}=0.01, k_{2}=k_{3}=k_{4}=k_{5}=1, \lambda=0.3, \mu=\nu=1$.

To summarize, we proposed a clustering model for the growth of a chiral crystal composed of achiral elements, and studied the conditions under which chiral symmetry breaking takes 
place. The dimers are assumed to be the smallest size possessing chiralities, and at the same time of the critical size. When the cluster size increases indefinitely by successive incorporation of achiral elements, a racemic mixture results.

In experiments, chirality selection is achieved by continuous stirring such that large crystals are fragmented into small clusters and the scattered small clusters induce secondary nucleation. In order to simulate the effect of the fragmentation caused by stirring, we introduce a decomposition process of large clusters into dimers in our crystallization model. If we restrict the cluster growth only up to the tetramers, the chiral symmetry is never broken. The result is interpreted in the quasi-steady approximation where clusters larger than the critical size are assumed to evolve sufficiently rapidly to attain the steady-state concentration. The approximation leads to the reduced rate equation of dimers with a linear autocatalysis, which is shown to be insufficient for breaking the chiral symmetry, as reported in our previous study. ${ }^{3}$

To equip the the model with the ability to break chiral symmetry dynamically, one needs a nonlinear autocatalytic process in the reduced rate equation of the dimers. This is acomplished by incorporating dimers into the other clusters to facilitate cluster growth. The process leads effectively to the nonlinear enhancement of the dimer production rate, or a nonlinear autocatalytic effect. We know from our previous study that the chiral symmetry can break in appropriate situations. ${ }^{3-5}$ Numerical integration of the full rate equations confirms this expectation. In the present model we included the coagulation of two dimers into a tetramer. This process consumes the minimum chiral units or dimers in a nonlinear way, but the fragmentation of a larger cluster, hexamer in our present case, replenishes dimers again into the crystallization process. If we incorporate dimer coagulation with clusters of larger sizes as $\mathrm{R}_{2}+\mathrm{R}_{j} \rightarrow \mathrm{R}_{j+2}$ and the breaking processes $\mathrm{R}_{j+2} \rightarrow \mathrm{R}_{2}+\mathrm{R}_{j}$, the parameter region for the chiral symmetry breaking is affected but the qualitative feature remains valid. In the present model, the dissociation which corresponds to the back reaction is again found essential, since without it the system relaxes to the fixed line in $r_{\Sigma}-s_{\Sigma}$ phase space, and no precise chirality selection takes place.

Thus far we have assumed that the smallest clusters, dimers, which show chirality are at the same time critical clusters which can dissociate, for mathematical simplicity. Actually there should be a margin between the two sizes, and the fragmentation of large clusters into those with sizes smaller than the critical ones might be the minimal requirement to sustain symmetry breaking. This aspect will be studied in the future.

\section{Acknowledgement}

Authors acknowledge support from the Gakuji-Shinkou-Shikin by Keio University. 


\section{References}

1) B. L. Feringa and R. A. van Delden: Angew. Chem. Int. Ed. 38 (1999) 3418.

2) F. C. Frank: Biochim. Biophys. Acta 11 (1953) 459.

3) Y. Saito and H. Hyuga: J. Phys. Soc. Jpn 73 (2004) 33.

4) Y. Saito and H. Hyuga: J. Phys. Soc. Jpn 73 (2004) 1685.

5) Y. Saito and H. Hyuga: in Progr. Chem. Phys. Res., ed. F. Columbus (Nova Science, New York, 2005) to appear.

6) K. Soai, T. Shibata, H. Morioka and K. Choji: Nature 378 (1995) 767.

7) I. Sato, D. Omiya, H. Igarashi, K. Kato, Y. Ogi, K. Tsukiyama and K. Soai: Tetrahedron Asymmetry 14 (2003) 975.

8) D. K. Kondepudi and K. Asakura: Acc. Chem. Res. 34 (2001) 946.

9) D. K. Kondepudi, R.J. Kaufman, and N. Singh: Science 250 (1990) 975.

10) C. Viedma: http://arXiv.org/abs/cond-mat./0407479.

11) M. Uwaha: J. Phys. Soc. Jpn. 73 (2004) 2601. 